\title{
Fuzzy term-rewriting system
}

\section{Churn Jung Liau and Bertrand I-peng Lin}

Department of Computer Science and Information Engineering, National Taiwan University, Taipei, Taiwan

Received March 1989

Revised January 1990

Abstract: This paper provides an approach to treat fuzzy equality in first order logic, specially in a theory in which only equality occurs as a predicate symbol (namely equational theory). Fuzzy term rewriting systems (FRS), that is the set of left to right directional equations, are also discussed. Finally, a fuzzy recognition algorithm is presented by applying FRS, which shows its potential use.

Keywords: Fuzzy logic; term rewriting; pattern recognition.

\section{Introduction}

In first order logic, the equality predicate plays an important role because 'equal to' is an essential relation in mathematics; specially in a theory in which only equality occurs as a predicate symbol (namely equational theory). For the purpose of computation, an equational theory can sometimes be reformulated as a term-rewriting system which provides an effective mechanism for equational reasoning. Recently a new approach to theorem proving in first order logic with equality based on the term rewriting method was proposed $[7,8]$ and has been shown to be as powerful as paramodulation and resolution combined. In other words, by means of algebraic logic, the rewriting method can be extensively used in logical inference. However, humans always use fuzzy reasoning to deal with the real world. Because of the extensive use of the term rewriting system, it is worthwhile to explore the possibility of embedding fuzzy inference within it.

For our purpose, we first generalize the concept of equality to fuzzy logic. Then, with the result of fuzzy equality, we define the fuzzy rewriting system and its inference mechanism, and finally, we discuss the possible applications and give a brief conclusion.

\section{Fuzzy equality}

In two-valued logic, for an interpretation $I$ over a fixed domain $D$, we mean a correspondence which assigns an $n$-ary operation in $D$ to each $n$-place function symbol, and an $n$-ary relation of $D$ to each $n$-place predicate symbol. Given an interpretation $I$ over $D$, we have the corresponding evaluation procedure $T$ to compute the truth value of each sentence (closed formula). Now, an inter- 
pretation $I$ is called an $E$-interpretation (of two-valued logic) if its evaluation procedure $T$ satisfies the following conditions:

(a) $T(t=t)=1$ for any ground term;

(b) If $T(s=t)=1$ and $T(\varphi[s])=1$, then $T(\varphi[t])=1$, where $s$ and $t$ are any ground terms, $\varphi[s]$ is any sentence and $\varphi[t]$ arises from $\varphi[s]$ by replacing one occurrence of $s$ by $t$.

In colloquial language, (b) states that if $s=t$ and $\varphi[s]$ holds, then $\varphi[t]$ holds too.

Let $s, t, \varphi[s]$ and $\varphi[t]$ be defined as above, then (b) can be restated as:

$\left(\mathrm{b}^{\prime}\right)$ If $T(s=t)=1$ then $T((\varphi[s] \& \sim \varphi[t]) \vee(\varphi[t] \& \sim \varphi[s]))=0$, which says that if $s=t$ holds then it is not possible that $\varphi[s]$ holds but $\varphi[t]$ does not or that $\varphi[t]$ holds but $\varphi[s]$ does not.

$\left(\mathrm{b}^{\prime \prime}\right)$ If $T(s=t)=1$ then $T(\varphi[s])=T(\varphi[t])$, which states that if $s=t$ holds then $\varphi[s]$ holds iff $\varphi[t]$ does.

Although (b), $\left(b^{\prime}\right)$ and $\left(b^{\prime \prime}\right)$ are equivalent in two-valued logic, when generalizing to fuzzy logic, we will see that they introduce quite different results-they interpret the equality symbol as three distinct fuzzy relations.

Definition 1 [11]. Let $D$ be any domain. Then an $n$-ary fuzzy relation $R$ in $D^{n}$ is a mapping from $D^{n}$ to the interval $[0,1]$.

An interpretation $I$ for fuzzy logic consists of an non-empty set $D$, called the domain of $I$ and a correspondence which assigns an $n$-ary fuzzy relation $P^{I}$ to each $n$-ary predicate symbol $P$ and an $n$-place operation $f^{l}$ in $D^{n}$ to each $n$-ary function symbol $f$. We will use $\left\langle D, P_{i}^{I}, f_{j}^{I}\right\rangle$, where $i, j$ are elements of some index sets, to denote the structure of the interpretation $I$. Given an interpretation $I$ over $D$, we have the corresponding evaluation procedure $T$ for each formula $\varphi$ in fuzzy logic as follows [13]:

(1) $T(\varphi)=P^{I}\left(d_{1}, \ldots, d_{n}\right)$, if $\varphi=P\left(t_{1}, \ldots, t_{n}\right)$ is a ground atom and each $t_{i}$ is evaluated to the domain element $d_{i}$ according to $l$.

(2) $T(\varphi)=1-T(\psi)$, if $\varphi=\sim \psi$.

(3) $T(\varphi)=\min \left\{T\left(\varphi_{1}\right), T\left(\varphi_{2}\right)\right\}$, if $\varphi=\varphi_{1} \& \varphi_{2}$.

(4) $T(\varphi)=\max \left\{T\left(\varphi_{1}\right), T\left(\varphi_{2}\right)\right\}$, if $\varphi=\varphi_{1} \vee \varphi_{2}$.

(5) $T(\varphi)=\inf \{T(\psi(a)) \mid a \in D\}$, if $\varphi=\forall x \psi(x)$.

(6) $T(\varphi)=\sup \{T(\psi(a)) \mid a \in D\}$, if $\varphi=\exists x \psi(x)$.

For simplicity, an interpretation for fuzzy logic is called a fuzzy interpretation.

Now, since Lee's evaluation procedure does not specify the semantics of the equality symbol, the natural question arises: what is the semantic meaning of the equality symbol in fuzzy logic?

Observing the two-valued case, we find that any evaluation procedure that satisfies (a), (b) (or (a), $\left(b^{\prime}\right)$, or $\left.(a)\left(b^{\prime \prime}\right)\right)$ will interpret the equality symbol as a congruence relation on domain $D$ (i.e. an equivalence relation satisfies substitution and replacement). However, in what follows, we will find that when generalized to fuzzy equality, the three equivalent ways to state (b) will result in three totally different fuzzy relations as the interpretation of fuzzy equality. After presenting the three generalizations, we will compare the difference between them, and point out which is the most suitable interpretation of fuzzy equality. 


\subsection{First generalization}

First, we extend Lee's evaluation procedure to include the semantics of the equality symbol by generalizing (a) and (b). If $s$ and $t$ are ground terms and $\varphi[s]$ and $\varphi[t]$ are defined as in (b), then we have

Definition 2. A fuzzy interpretation $I$ over $D$ is called an $E$-interpretation if the evaluation procedure $T$ with respect to $I$ satisfies the following conditions:

(7) $T(t=t)=1$.

(8) $\min \{T(s=t), T(\varphi[s])\} \leqslant T(\varphi[t])$.

Note that (7), (8) imply (a), (b) respectively.

Because our aim is to explore the possibility of combining fuzzy reasoning with the rewriting system, we first need to investigate the influence of fuzziness on them. In the following context, $T$ is an evaluation procedure with respect to an $E$-interpretation.

Proposition 1. Let $s, t, u$ be terms. Then

(i) Symmetry: $T(s=t)=T(t=s)$,

(ii) Transitivity: $T(s=u) \geqslant \min \{T(s=t), T(t=u)\}$,

(iii) Substitution: $T(s \sigma=t \sigma) \geqslant t(s=t)$ where $\sigma$ is any substitution,

(iv) Replacement: $T(u[s]=u[t]) \geqslant T(s=t)$, where $u[s]$ is a term containing a subterm $s$, and $u[t]$ is the result of replacing some occurrences of $s$ in $u[s]$ by $t$.

Proof. (i) We prove it first in the case where $s$ and $t$ are ground terms. By Definition 2, we have

$$
\begin{aligned}
& \min \{T(s=t), T(s=s)\} \leqslant T(t=s), \\
& \min \{T(t=s), T(t=t)\} \leqslant T(s=t) .
\end{aligned}
$$

Now since $T(s=s)=T(t=t)=1$,

$$
T(s=t) \leqslant T(t=s), \quad \text { and } \quad T(t=s) \leqslant T(s=t) .
$$

Hence $T(s=t)=T(t=s)$.

For non-ground terms $s$ and $t$, we have

$$
\begin{aligned}
T(s=t) & =\inf \{T(s=t) \sigma \mid \sigma \text { is ground substitution }\} \\
& =\inf \{T(t=s) \sigma \mid \sigma \text { is ground substitution }\} \\
& =T(t=s) .
\end{aligned}
$$

(ii) When $s, t$ and $u$ are ground terms, we obtain the result directly from Definition 2. For the general case, we can get the result,

$$
\begin{aligned}
& T(s=t)=\inf _{\sigma}\{T((s=t) \sigma) \mid \sigma \text { is any ground substitution }\} \\
& =\inf _{\sigma}\{T(s \sigma=t \sigma)\} \\
& \geqslant \min \left\{\inf _{\sigma}\{T(s \sigma=t \sigma)\}, \inf \{T(t \sigma=u \sigma)\}\right\} \\
& =\min \{T(s=t), T(t=u)\} .
\end{aligned}
$$


(iii) $\quad T(s \sigma=t \sigma)=\inf \{T(s \sigma \beta=t \sigma \beta) \mid \beta$ is any ground substitution $\}$

$$
\begin{aligned}
& \geqslant \inf \{T(s \gamma=t \gamma) \mid \text { is any ground substitution }\} \\
& =T(s=t) .
\end{aligned}
$$

(iv) If $s$ and $t$ are ground terms, then

$$
\min \{T(s=t), T(u[s]=u[s])\} \leqslant T(u[s]=u[t]) .
$$

Therefore

$$
T(u[s]=u[t]) \geqslant T(s=t) .
$$

It is easy to raise the result up to the general case as in (iii).

\subsection{Second generalization}

If we generalize the concept of fuzzy equality from (a) and ( $\left.b^{\prime}\right)$, then we get the following definition.

Definition 3. A fuzzy interpretation $I$ is called an $E^{\prime}$-interpretation, if the evaluation procedure $T^{\prime}$ of $I$ satisfies the following conditions:

(7') $T^{\prime}(t=t)>0.5$,

(8) $T^{\prime}((\varphi[s] \& \sim \varphi[t]) \vee(\varphi[t] \& \sim \varphi[s])) \leqslant 1-T^{\prime}(s=t)$, where $t, s, \varphi[t]$ and $\varphi[s]$ are defined as in Definition 2.

Here, we only require that $T^{\prime}(t=t)>0.5$, because if $T^{\prime}(t=t)=1$ for any ground term $t$, then $\left(8^{\prime}\right)$ reduces the system to two-valued logic.

Also, $\left(8^{\prime}\right)$ implies $\left(b^{\prime}\right)$, but $\left(7^{\prime}\right)$ implies (a) only when $T$ is restricted as a two-valued evaluation procedure.

We presented a result of this definition in a previous paper [14], and we restate it without proof in what follows.

Proposition 2. Let $T^{\prime}$ be an evaluation procedure with respect to an $E^{\prime}$ interpretation and $s, t, u, u[s], u[t]$ be the same as in Proposition 1. Then

(i) Symmetry: if $T^{\prime}(s=t)>0.5$ then $T^{\prime}(s=t)=T^{\prime}(t=s)$.

(ii) Transitivity: if $\min \left(T^{\prime}(s=t), T^{\prime}(t=u)\right)>0.5$, then $T^{\prime}(s=t)=T^{\prime}(t=u)$ $=T^{\prime}(s=t)$.

(iii) Substitution: $T(s \sigma=t \sigma) \geqslant T(s=t)$ where $\sigma$ is any substitution.

(iv) Replacement: if $T(s=t)>0.5$ then $T(u[s]=u[t]) \geqslant T(s=t)$.

\subsection{Third generalization}

Now, according to $(a),\left(b^{\prime \prime}\right)$, we have the following generalization.

Definition 4. An interpretation $I$ is called an $E^{\prime \prime}$-interpretation if the evaluation procedure $T^{\prime \prime}$ of $I$ satisfies the following conditions:

(7") $T^{\prime \prime}(t=t)=1$,

$\left(8^{\prime \prime}\right)\left|T^{\prime \prime}(\varphi[s])-T^{\prime \prime}(\varphi[t])\right| \leqslant 1-T^{\prime \prime}(s=t)$,

where $s, t, \varphi[s]$ and $\varphi[t]$ are as in Definition 2 . 
As above, $\left(7^{\prime \prime}\right),\left(8^{\prime \prime}\right)$ imply $(\mathrm{a}),\left(\mathrm{b}^{\prime \prime}\right)$ respectively.

Furthermore, $\left(8^{\prime \prime}\right)$ can be rewritten as $T^{\prime \prime}(s=t) \leqslant 1-\left|T^{\prime \prime}(\varphi[s])-T^{\prime \prime}(\varphi[t])\right|$, and the right-hand side is in fact the semantic evaluation of ' $\varphi[s] \equiv \varphi[t]$ ' in [5], but we will not dwell upon it, since in our evaluation procedure, we will not specify the semantic of logical equivalence symbol ' $\equiv$ '.

Similarly, we have the following result.

Proposition 3. Let $T^{\prime \prime}$ be an evaluation procedure of an $E^{\prime \prime}$-interpretation, and $s, t$, $u, u[s]$ and $u[t]$ be the same as in Proposition 1. Then, we have

(i) Symmetry: $T^{\prime \prime}(s=t)=T^{\prime \prime}(t=s)$,

(ii) Transitivity: $T^{\prime \prime}(s=u) \geqslant T^{\prime \prime}(s=t)+T^{\prime \prime}(t=u)-1$,

(iii) Substitution: $T^{\prime \prime}((s=t) \sigma) \geqslant T^{\prime \prime}(s=t)$ for any substitution $\sigma$,

(iv) Replacement: $T^{\prime \prime}(u[s]=u[t]) \geqslant T^{\prime \prime}(s=t)$.

Proof. The proof of (iii) is the same as that of Proposition 1, since the definition of fuzzy equality is irrelevant to it. For other parts, we only prove the ground case, and it is easy to raise them to the general case in the same way as in Proposition 1.

(i) From ( 8 "), we have

$$
\left|T^{\prime \prime}(s=s)-T^{\prime \prime}(s=t)\right| \leqslant 1-T^{\prime \prime}(t=s)
$$

and

$$
\left|T^{\prime \prime}(t=t)-T^{\prime \prime}(t=s)\right| \leqslant 1-T^{\prime \prime}(s=t) ;
$$

then from $\left(7^{\prime \prime}\right)$, the result follows.

(ii) From (i) and $\left(8^{\prime \prime}\right)$, we have

$$
\left|T^{\prime \prime}(t=u)-T^{\prime \prime}(s=u)\right| \leqslant 1-T^{\prime \prime}(s=t)
$$

and so

$$
T^{\prime \prime}(s=u) \geqslant T^{\prime \prime}(s=t)+T^{\prime \prime}(t=u)-1 .
$$

(iii) From $\left(8^{\prime \prime}\right)$, we have

$$
\left|T^{\prime \prime}(u[s]=u[s])-T^{\prime \prime}(u[s]=u[t])\right| \leqslant 1-T^{\prime \prime}(s=t)
$$

and so, we obtain

$$
T^{\prime \prime}(u[s]=u[t]) \geqslant T^{\prime \prime}(s=t), \quad \text { by }\left(7^{\prime \prime}\right) .
$$

\subsection{The comparison}

From the viewpoint of syntax, the equality predicate is merely a meaningless symbol, and in two-valued logic, we interpreted it as a congruence relation for practical needs. Now, according to the above discussion, we have three different evaluation procedures $T, T^{\prime}$ and $T^{\prime \prime}$ which correspond to three different fuzzy relations:

$T$ : satisfies conditions (1)-(8) (and so Proposition 1),

$T^{\prime}$ : satisfies conditions (1)-(6), $\left(7^{\prime}\right),\left(8^{\prime}\right)$ (and so Proposition 2$)$,

$T^{\prime \prime}$ : satisfies conditions (1)-(6), $\left(7^{\prime \prime}\right),\left(8^{\prime \prime}\right)$ (and so Proposition 3$)$.

Then, how do we choose among them? 
First, the transitivity property plays the role of chaining in equational reasoning, so from Proposition 3, if we adopt the third generalization, some results inferred by the chaining may be trivial. For example, let $s, t$ and $u$ be defined as in Proposition 3, then we can get the trivial result $T^{\prime \prime}(s=u) \geqslant 0$ from $T^{\prime \prime}(s=t) \geqslant 0.7$ and $T^{\prime \prime}(t=u) \geqslant 0.3$. Therefore, for technical considerations, we discard $E^{\prime \prime}$-interpretation as the semantic meaning of the equality predicate in fuzzy logic.

Between the remaining two candidates, $T$ seems more practical than $T^{\prime}$ from the following definition and the critical difference between their transitivities.

Definition 5 [11]. A similarity relation, $S$, is a fuzzy relation in $X$ such that for all $x, y, z \in X$,

$$
\begin{aligned}
& S(x, x)=1, \\
& S(x, y)=S(y, x), \\
& S(x, z) \geqslant \max _{y \in X}[\min (S(y, z))] .
\end{aligned}
$$

$T$ interprets the equality symbol as not only a similarity relation, but also a fuzzy congruence relation in the following sense.

Definition 6. A fuzzy relation $C$ in $X$ is a fuzzy congruence relation if $C$ is a similarity relation and for any operation $f$ in $X$ and $x, y \in X$, we have $C(f(\ldots, x, \ldots), f(\ldots, y, \ldots) \geqslant C(x, y)$.

From these definitions, we find that $T$ interprets fuzzy equality as a fuzzy congruence relation naturally, but $T^{\prime}$ does not have this property.

Furthermore, more oddly, if we change the reflexivity $\left(7^{\prime}\right)$ into $T^{\prime}(t=t)=1$, then $\left(8^{\prime}\right)$ will force our system to reduce to 2-valued logic; but according to Kandel's definition, the reflexivity must be definitely true for a similarity relation.

Therefore, though we can take any one of the three fuzzy relations as the semanitical meaning of fuzzy equality, the one corresponding to $T$ is doubtless the most suitable.

Note. The oddness of $T^{\prime}$ lies mainly in that $\left(\mathrm{b}^{\prime}\right)$ writes ' $\varphi[s] \equiv \varphi[t]$ ' as $'(\sim \varphi[s] \vee \varphi[t]) \&(\sim \varphi[t] \vee \varphi[s])$ ' which violates the principle of entailment when generalized to fuzzy logic. (We are thankful that the referees pointed this out to us). However, even if we adopt $T^{\prime}$ as the evaluation procedure of fuzzy logic with equality, it does not logically imply that we must adopt $\min \left(\max \left(1-T^{\prime}(p)\right.\right.$, $\left.\left.T^{\prime}(q)\right), \max \left(1-T^{\prime}(q), T^{\prime}(p)\right)\right)$ as the definition of $T^{\prime}(p \equiv q)$. Therefore, theoretically, we can still adopt $T^{\prime}$ as an evaluation procedure of fuzzy logic with equality without violating the principle of entailment, though it seems strange and impractical. 


\section{Fuzzy term-rewriting system}

Given an equational theory $E$, we can form an equivalent term rewriting system $R=\left\{l_{j} \rightarrow r_{j} \mid j \in J\right\}$ such that $s=t$ is true in $\left\{l_{j}=r_{j} \mid j \in J\right\}$ iff $s=t$ is true in $E$, where $J$ is a finite index set. We seek to incorporate the concept of fuzzy equality into the term rewriting system in this section.

A fuzzy rewriting system FRS is a set of ordered pairs $\left\{\left(l_{j} \rightarrow r_{j}, \alpha_{j}\right) \mid j \in J\right\}$, where $J$ is a finite set, $l_{j} \rightarrow r_{j}$ is an ordinary rewriting rule, and $\alpha_{j} \in(0,1]$. Each pair is called a fuzzy rewriting rule. A term $t$ is reduced using the rule $(1 \rightarrow r, \alpha)$ if a subterm $s$ of $t$, which is an instance of the left-hand side $l$, is replaced by the corresponding instance of $r$. A term $s$ is reachable from $t$ if $t$ can be reduced to $s$ after a finite number of reductions. A term is irreducible if no rule can be applied to it. More definitions, such as termination and confluence, are the same as in the ordinary rewriting system [10].

Definition 7. A fuzzy algebra accompanied by an FRS $\left\{\left(l_{j} \rightarrow r_{j}, \alpha_{j}\right) \mid j \in J\right\}$ is the structure of a fuzzy $E$-interpretation $I$, where the evaluation procedure $T$ of $I$ satisfies $T\left(l_{j}=r_{j}\right) \geqslant \alpha_{j}$ for all $j \in J$.

The variety of an FRS is the set of all fuzzy algebras accompanying it.

Notation. Let $V$ be the variety of an FRS $\left\{\left(l_{j} \rightarrow r_{j}, \alpha_{j}\right) \mid j \in J\right\}$. We write $V F(s=t, \alpha)$ if for any evaluation procedure $T$ of an $E$-interpretation such that $T\left(l_{j}=r_{j}\right) \geqslant \alpha_{j}$ for all $j \in J$, we have $T(s=t) \geqslant \alpha$.

In the above definitions, we borrowed some terminology from universal algebra. For their original meaning, see ([2]).

Now, we modify the concept of critical pairs, the key idea of the Knuth-Bendix procedure [12], to meet our definition.

From now on, we use $t[u]$ to denote that $u$ is a subterm of $t$, and $t^{*}$ to denote an irreducible form of $t$.

Definition 8. (i) Given an FRS $R$ and two rules $\left(l_{1} \rightarrow r_{1}, \alpha_{1}\right),\left(l_{2}[u] \rightarrow r_{2}, \alpha_{2}\right)$ in $R$ where $u$ is not a variable, if there is an mgu $\sigma$ such that $l_{1} \sigma=u \sigma$, then $\left\langle\left(l_{2}\left[r_{1}\right] \sigma, r_{2} \sigma\right), \min \left(\alpha_{1}, \alpha_{2}\right)\right\rangle$ is a critical pair of the two rules. A critical pair is divergent if $\left(l_{2}\left[r_{1}\right] \sigma\right)^{*} \neq\left(r_{2} \sigma\right)^{*}$.

(ii) A critical pair $\langle(s, t), \alpha\rangle$ is said to be simplified by a rule $(l \rightarrow r, \beta)$, if $s$ (or $t$ ) is reduced to $s^{\prime}$ (resp. $t^{\prime}$ ) by $l \rightarrow r$ in the sense of an ordinary rewriting system, and the resulting pair is $\left\langle\left(s^{\prime}, t\right), \min (\alpha, \beta)\right\rangle$ (resp. $\left.\left\langle\left(s, t^{\prime}\right), \min (\alpha, \beta)\right\rangle\right)$.

(iii) A rule $\left(l_{1} \rightarrow r_{1}, \alpha_{1}\right)$ is reduced by $\left(l_{2} \rightarrow r_{2}, \alpha_{2}\right)$ to $\left(l_{1} \rightarrow r_{1}, \min \left(\alpha_{1}, \alpha_{2}\right)\right)$, if $r_{1}$ is reduced to $r_{1}^{\prime}$ by $l_{2} \rightarrow r_{2}$.

(iv) Let $R=\left\{\left(l_{j} \rightarrow r_{j}, \alpha_{j}\right) \mid j \in J\right\}$ be an FRS. If $(l \rightarrow r, \alpha) \in R$, then we write $(l \rightarrow r, \alpha) \Rightarrow\left(l \rightarrow r^{*}, \alpha^{*}\right)$ when $(l \rightarrow r, \alpha)$ is right-reduced to $\left(1 \rightarrow r^{*}, \alpha^{*}\right)$ by repetitive use of the other rules of $R$.

From Proposition 1 and the above definition, we have the following result. 
Lemma 4. Let $R=\left\{\left(l_{j} \rightarrow r_{j}, \alpha_{j}\right) \mid j \in J\right\}$ be an FRS and $T$ be an evaluation procedure of a fuzzy E-interpretation such that $T\left(l_{j}=r_{j}\right) \geqslant \alpha_{j}$ for all $j \in J$. Then

(i) if $\langle(s, t), \alpha\rangle$ is a critical pair, then $T(s=t) \geqslant \alpha$.

(ii) if $\langle(s, t), \alpha\rangle$ is a critical pair and $\left\langle\left(s^{\prime}, t\right), \alpha^{\prime}\right\rangle$ is a simplified form of $\langle(s, t), \alpha\rangle$, then $T\left(s^{\prime}=t\right) \geqslant \alpha^{\prime}$.

(iii) if $\left(l_{i} \rightarrow r_{i}, \alpha_{i}\right) \in R$ is right-reduced to $\left(l_{i} \rightarrow r_{r}^{\prime}, \alpha_{i}^{\prime}\right)$ using a rule of $R$, then $T\left(l_{i}=r_{i}^{\prime}\right) \geqslant \alpha_{i}^{\prime}$.

Proof. (i) From the assumption, we have

$$
T\left(l_{1}=r_{1}\right) \geqslant \alpha_{1} \text { and } T\left(l_{2}[u]=r_{2}\right) \geqslant \alpha_{2} .
$$

So by substitution, we have

$$
\begin{aligned}
T\left(l_{2}\left[l_{1}\right] \sigma=r_{2} \sigma\right) & =T\left(l_{2}[u] \sigma=r_{2} \sigma\right) \\
& \geqslant T\left(l_{2}[u] \sigma=r_{2}\right) \geqslant \alpha_{2} .
\end{aligned}
$$

Now, using replacement, we have

$$
T\left(l_{2}\left[l_{1}\right]=l_{2}\left[r_{1}\right]\right) \geqslant T\left(l_{1}=r_{1}\right) \geqslant \alpha_{1} .
$$

Hence

$$
T\left(l_{2}\left[l_{1}\right] \sigma=l_{2}\left(r_{1}\right] \sigma\right) \geqslant \alpha_{1} .
$$

Finally, from transitivity and symmetry, we have

$$
T\left(l_{2}\left[r_{1}\right] \sigma=r_{2} \sigma\right) \geqslant \min \left(\alpha_{1}, \alpha_{2}\right) .
$$

(ii) From (i), $T(s=t) \geqslant \alpha$ and from the assumption, $T(l=r) \geqslant \beta$. Now by substitution, we have

$$
T(s=r \sigma)=T(l \sigma=r \sigma) \geqslant \beta \text { for any } \sigma \text { such that } s=l \sigma .
$$

By, transitivity and symmetry, we have $T(r \sigma=t) \geqslant \min (\alpha, \beta)$, i.e., $T\left(s^{\prime}=t\right) \geqslant \alpha^{\prime}$.

(iii) If $\left(l_{i} \rightarrow r_{i}, \alpha_{i}\right)$ is reduced by $\left(l_{k} \rightarrow r_{k}, \alpha_{k}\right)$, then, from the assumption, we have

$$
T\left(l_{i}=r_{i}\right) \geqslant \alpha_{i}, \quad T\left(l_{k}=r_{k}\right) \geqslant \alpha_{k} .
$$

Now, following a similar argument as in (ii), we obtain

$$
T\left(l_{i}=r_{i}^{\prime}\right) \geqslant \alpha_{i}^{\prime} .
$$

Now, we can state the word problem in the fuzzy algebra formally and give an algorithmic solution to it.

Fuzzy word problem. Given an FRS, and two terms $t_{1}, t_{2}$, we seek to find $\alpha$ such that $V \vDash\left(t_{1}=t_{2}, \alpha\right)$, where $V$ is the variety of the FRS. 
Next, we modify the Knuth-Bendix algorithm to give a solution to our problem. For simplicity, we adopt a short version from [1], and separate it into two phases, i.e. the completion phase and the decision phase.

\section{Algorithm for fuzzy word problem.}

Inputs: An FRS $R=\left\{\left(l_{j} \rightarrow r_{j}, \alpha_{j}\right) \mid 1 \leqslant j \leqslant n\right\}$, two terms $t_{1}$ and $t_{2}$, and a finite termination ordering " $>$ " (see [3]).

Output: A number ans $\in(0,1]$ such that $V \vDash\left(t_{1}=t_{2}\right.$, ans $)$ where $V$ is the variety of $R$.

\section{Steps:}

I. COMPLETION PHASE

begin

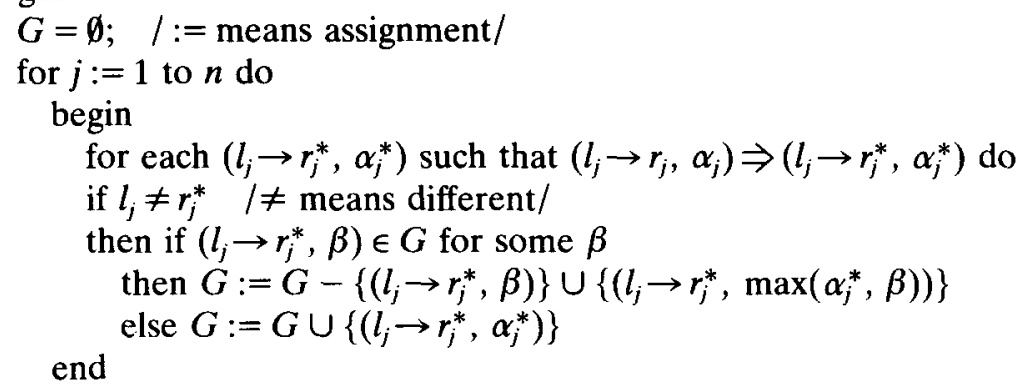

while there are divergent critical pairs from $G$ do

begin

find a divergent critical pair $\langle(s, t), \alpha)\rangle$;

simplify it into irreducible form $\left\langle\left(s^{*}, t^{*}\right), \alpha^{*}\right\rangle$ in the sense of Def 8;

if $s^{*}>t^{*}$

then

if $\left(s^{*} \rightarrow t^{*}, \beta\right) \in G$ for some $\beta$

then $G:=G-\left\{\left(s^{*} \rightarrow t^{*}, \beta\right)\right\} \cup\left\{\left(s^{*} \rightarrow t^{*}, \max \left(\alpha^{*}, \beta\right)\right)\right\}$

else begin

$$
G:=G \cup\left\{\left(s^{*} \rightarrow t^{*}, \alpha^{*}\right)\right\} ;
$$

right-reduce (or delete) other rules of $G$ using $\left(t^{*} \rightarrow s^{*}, \alpha^{*}\right)$ end

else if $t^{*}>s^{*}$

then if $\left(t^{*} \rightarrow s^{*}, \beta\right) \in G$ for some $\beta$

then $G:=G-\left\{\left(t^{*} \rightarrow s^{*}, \beta\right)\right\} \cup\left\{\left(t^{*} \rightarrow s^{*}, \max \left(\alpha^{*}, \beta\right)\right)\right\}$

else begin

$$
G:=G \cup\left\{\left(t^{*} \rightarrow s^{*}, \alpha^{*}\right)\right\} ;
$$

right-reduce (or delete) other rules of $G$ using $\left(t^{*} \rightarrow s^{*}, \alpha^{*}\right)$ end

else abort

end 
II. Decision Phase

begin

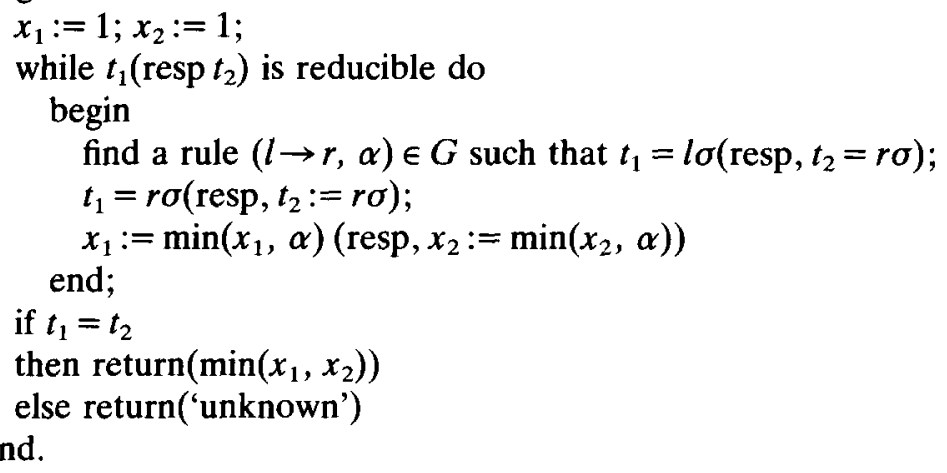

Observing the above alogrithm, one can find that it is nondeterministic, so we can only get the lower bound of $T\left(t_{1}=t_{2}\right)$, not necessary the greatest one. We can give an example to show this.

Example. Inputs:

(1)

$$
\begin{aligned}
& R=\{1 .(f(g(x), h(a)) \rightarrow g(x), 0.7) \\
& \text { 2. }(f(g(a), h(x)) \rightarrow h(x), 0.5) \\
& \text { 3. }(g(a) \rightarrow a, \quad 0.4) \\
& \text { 4. }(h(a) \rightarrow a, \quad 0.6)\} \text {, }
\end{aligned}
$$

where $a$ is constant symbol and $x$ is a variable.

(2) Two terms:

$$
t_{1}=f(g(a), h(a)), \quad t_{2}=a .
$$

(3) An ordering on terms such that $t[s]>s$ for each term $t$ and subterm $s$ of $t$. Obviously, the ordering is finite terminating from [3].

Then after execution of the COMPLETION PHASE

$$
\begin{aligned}
& G=\{1 . f(g(x), h(a)) \rightarrow g(x), 0.7) \\
& \text { 2. }(f(g(a), h(x)) \rightarrow h(x), 0.5) \\
& \text { 3. }(g(a) \rightarrow a, \quad 0.4) \\
& \text { 4. }(h(a) \rightarrow a, \quad 0.6) \\
& \text { 5. }(f(g(x), a) \rightarrow g(x), \quad 0.6) \text { /from } 1,4 / \\
& \text { 6. }(f(a, h(x)) \rightarrow h(x), \quad 0.4) \quad / \text { from } 2,3 / \\
& \text { 7. }(f(a, h(a)) \rightarrow a, \quad 0.4) \quad / \text { from } 1,3 / \\
& \text { 8. }(f(g(a), a) \rightarrow a, \quad 0.5) \text { /from } 2,4 / \\
& \text { 9. }(f(a, a) \rightarrow a, \quad 0.4) \quad / \text { from } 3,5 \text { or } 3,8 \text { or } 4,6 \text { or } 4,7 /\} \text {. }
\end{aligned}
$$


When executing the Desision PHASE there are at least two possible returned values:

(i) From rules 1 and 3

$$
t_{1}=f(g(a), h(a)) \stackrel{0.7}{\longrightarrow} g(a) \stackrel{0.4}{\longrightarrow} a=t_{2}
$$

and returned was 0.4 .

(ii) From rules 2 and 4

$$
t_{1} f(g(a), h(a)) \stackrel{0.5}{\longrightarrow} h(a) \stackrel{0.6}{\longrightarrow} a=t_{2}
$$

and returned was 0.5 .

Now, we show the correctness of this algorithm. First the following soundness lemma is given.

Lemma 5. Let $R=\left\{\left(l_{j} \rightarrow r_{j}, \alpha_{j}\right) \mid j \in J\right\}$ be an FRS. If $T$ is an evaluation procedure of an E-interpretation such that $T\left(l_{j}=r_{j}\right) \geqslant \alpha_{j}$ for all $j \in J$, then for any rule $(s \rightarrow t, \alpha) \in G$, we have $T(s=t) \geqslant \alpha$ in any time.

Proof. By repetitive use of Lemma 4, we can get the result immediately.

Theorem 6 (Partial correctness of algorithm). Given an FRS $R=\left\{\left(l_{j} \rightarrow\right.\right.$ $\left.\left.r_{j}, \alpha_{j}\right) \mid j \in J\right\}$, let $V$ be the variety of $R$. If $t_{1}=t_{2}$ is a logical consequence of the equational theory $\left\{l_{j}=r_{j} \mid j \in J\right\}$, then when the algorithm terminates, we have $V \vDash\left(t_{1}=t_{2}\right.$, ans $)$, where ans is the return value.

Proof. By the assumption and the correctness of the ordinary Knuth-Bendix procedure, Decision PHASE will succeed (i.e. $t_{1}$ and $t_{2}$ will be reduced to the same term), and by Lemma 5 and Proposition 1, we have

$$
T\left(t_{1}=t_{1}^{\prime}\right) \geqslant x_{1}, \quad T\left(t_{2}=t_{2}^{\prime}\right) \geqslant x_{2} .
$$

After the exit of while-loop in Decision PHASE, by Proposition 1 (symmetry and transitivity), we have

$$
T\left(t_{1}=t_{2}\right) \geqslant \min \left(x_{1}, x_{2}\right)=\text { ans, }
$$

for each evaluation procedure $T$ such that $T\left(l_{j}=r_{j}\right) \geqslant \alpha_{j}$ for all $j \in J$, i.e. $V \vDash\left(t_{1}=t_{2}\right.$, ans $)$.

Just like the ordinary Knuth-Bendix procedure, there are three possible results for our algorithm, i.e. succeed, abort, or loop forever. To avoid the abort condition, it should be easy to generalize our algorithm to Hsiang's Unfailed Knuth-Bendix procedure [8].

\section{Applications}

In this section, we will present two possible applications of FRS. The first serves as a tool for fuzzy inference, and the other one is applied to the pattern recognition problem. 


\subsection{FRS as a tool of fuzzy inference}

Here, we discuss the possibility of inferring the fuzzy truth of a formula from a set of axioms in propositional fuzzy logic by FRS.

First, we introduce the axioms of Boolean algebra BA [4].

$$
\begin{aligned}
\mathrm{BA}=\{(x+y)+z=x+(y+z),(x y) z=x(y z) \text { (associativity laws), } & \\
& x+y=y+x, x y=y x \text { (commutativity laws), } \\
& x+(y z)=(x+y)(x+z), x(y+z)=x y+x z \text { (distributivity laws), } \\
& -(x+y)=(-x),-(x y)=-x+(-y) \text { (De Morgan's laws), } \\
& x+x=x, x x=x \text { (idempotency laws), } \\
& -(-x)=x \text { (double negation law), } \\
& x+(x y)=x, x(x+y)=x \text { (absorption laws), } \\
& x+0=x, x 1=x, x+1=1, x 0=0 \text { (laws of zero and one), } \\
& x+(-x)=1, x(-x)=0 \text { (exclude-middle laws) }\}
\end{aligned}
$$

Then, we have the following well-known lemma to provide a link between the inference of propositional logic and equational reasoning of Boolean algebra.

Lemma 7. If $S$ is a set of formulas and $\varphi$ is its logical consequence, then $\varphi^{\prime}=1$ is a logical consequence of $\mathrm{BA} \cup\left\{\psi^{\prime}=1 \mid \psi^{\prime} \in S\right\}$, where $\varphi^{\prime}$ and $\psi^{\prime}$ are the corresponding Boolean expressions of $\varphi$ and $\psi$ respectively (i.e. change $\sim, \vee, \&$ into,,$-+ \cdot$ respectively in the original formula).

Now, let $F_{\mathrm{BA}}=\{(l \rightarrow r, 0.5) \mid l=r$ is the exclude-middle laws $\} \cup\{(l \rightarrow r, 1) \mid$ $l=r$ is one of the other laws in BA $\}$. Suppose that $S$ is a set of formulas, and $S^{\prime}$ is the corresponding set of Boolean expressions. Then, we can define an FRS $R(S)=F_{\mathrm{BA}} \cup\left\{\left(\varphi^{\prime} \rightarrow 1, a\right) \mid \varphi^{\prime} \in S^{\prime}, T\left(\varphi^{\prime}=1\right) \geqslant a\right\}$ according to some evaluation procedure $T$. (Of course, $T$ depends on the real world situation and what the formula $\varphi$ represents).

Then, given $S$ and another formula $\psi$, we can take $R(S), \psi^{\prime}, 1$ as the three inputs to our algorithm for the fuzzy word problem, and get the result $T\left(\psi^{\prime}=1\right) \geqslant$ ans as specified by the algorithm.

In other words, given a set of (fuzzy) premises, if we can know the truth value of sentences like ' $\varphi$ is true' (where $\varphi$ is a premise), then given any formula $\psi$, we can get the truth value of ' $\psi$ is true'.

\subsection{Fuzzy recognition}

Conceptually, an object is recognized as another one if they possess some similarities, and since Definition 5 and Proposition 1 catch the intuitive idea of the similarlity relation, it is natural to apply fuzzy equality to pattern recognition problems.

Now, we can consider the following problem. 
When an object can be decomposed into small components, and each component has been recognized respectively, how can we apply FRS to recognize a combined object?

To solve it, we first give the following formal definition.

Definition 9. A fuzzy recognition system is a quadruple $\left\langle C_{1}, C_{2}, F, R\right\rangle$, where $C_{1}$ is a set of basic components, $C_{2}$ is another set of components. $F$ is a set of composing functions and $R$ is an FRS such that for each rule $(l \rightarrow r, \alpha) \in R$, $r \in \operatorname{Term}\left(C_{2}, F\right)$, and $l \in \operatorname{Term}\left(C_{1} \cup C_{2}, F\right) \backslash \operatorname{Term}\left(C_{2}, F\right)$, where $\operatorname{Term}(A, F)$ denotes the set of terms constructed by constant symbols in $A$ and function symbols in $F$.

Now, we can give the recognition algorithm as follows.

\section{Algorithm for fuzzy recognition system.}

Inputs: A fuzzy recognition system $\left\langle C_{1}, C_{2}, F, R\right\rangle$, a term $t \in T\left(C_{1}, F\right)$.

Outputs: A term $t^{\prime} \in T\left(C_{2}, F\right)$ and a number $\alpha \in(0,1]$ (or 'unknown').

\section{Steps:}

$$
\text { TYPE } \begin{aligned}
T_{1} & =\operatorname{Term}\left(C_{1} \cup C_{2}, F\right) ; \\
T_{2} & =\operatorname{Term}\left(C_{1} \cup C_{2}, F\right) \times[0,1] ;
\end{aligned}
$$

FUNCTION REC(term : $\left.T_{1}\right): T_{2} ;$ /term: object to be recognized/

$\operatorname{Var} x, m t, \operatorname{term} 1: T_{1} ; /(m t, m x)$ : the most possible result up to now/ $m x, y:[0,1]$

begin

if term is irreducible w.r.t. $R$

then if term $\in \operatorname{Term}\left(C_{2}, F\right)$

then return((term, 1)) /successfully recognized/

else return $(($ term, 0$))$; cannot be recognized/

$m x:=0$

for each rule $(l \rightarrow r, \alpha) \in R$ do

if term can be reduced to term 1 by $(l \rightarrow r) \quad /$ term is similar to term 1 then begin with fuzzy degree $\alpha /$

$(x, y):=\operatorname{REC}(\operatorname{term} 1) ; /$ term 1 is recognized as $x$ with degree $y /$

$y:=\min (\alpha, y) ; \quad /$ by transitivity, term is recognized as $x$ with degree $y /$

if $y>m x$ /if degree $y$ is larger than the most possible value up to now/

then begin /then update the most possible result up to now/

$$
\begin{aligned}
& m x:=y ; \\
& m t:=x
\end{aligned}
$$

end

end;

return $((m t, m x))$ /return the most possible result/ end. 


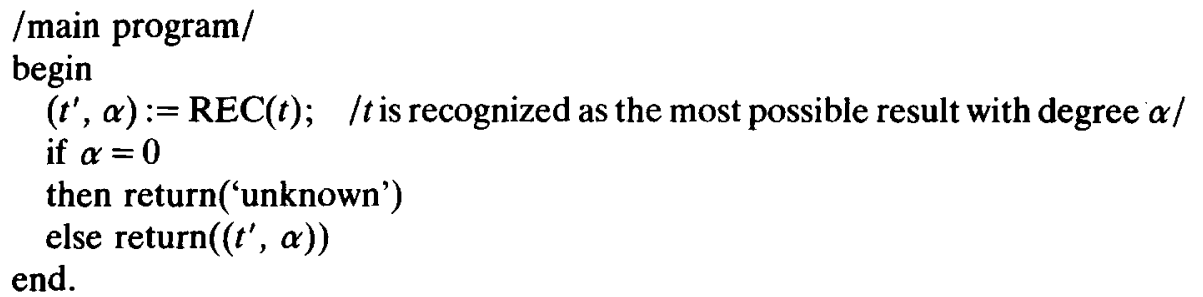

The above algorithm finds one of the terms $t^{\prime}$ such that $T\left(t=t^{\prime}\right)$ is as large as possible subject to the requirement of Definition 3 and $T(l=r) \geqslant \alpha$ for each rule $(l \rightarrow r, \alpha)$ in $R$. There may exist more than one answer for $t^{\prime}$, and we can even find all of them, but for efficiency, we adopt the present version.

According to the definition of the recognition system, when each recursive call of REC occurs, the argument contains less symbols in $C_{1}$, so the algorithm always terminates.

Finally, a term is said to recognizable in a fuzzy recognition system if the output of above algorithm is not 'unknown'.

\section{Conclusions}

We have proposed a concept of fuzzy equality which is theoretically interesting and catches the intuitive idea of the similarity relation.

Moreover, while the similarity relation is the fuzzy counterpart of the equivalence relation, our fuzzy equality is that of the congruence relation. Therefore, fuzzy equality should be potentially useful.

\section{References}

[1] B. Buchberger, Basic features and development of the critical-pair/completion procedure, in: 1st Conf. on Rewriting Technique and Application (1985).

[2] P.M. Cohn, Universal Algebra (D. Reidel, Dordrecht, 1981).

[3] N. Dershowitz, Orderings for term-rewriting systems, Theoret. Comput. Sci. 17 (1982) $279-301$.

[4] J.H. Gallier, Logic for Computer Science (Harper and Row, New York, 1986).

[5] B.R. Gaines, Fuzzy and probability uncertainty logics, Inform. and Control 38 (1978) 154-169.

[6] J. Hsiang, Refutational theorem proving using term-rewriting systems, Artificial Intelligence 25 (1985) 255-300.

[7] J. Hsiang, Rewrite method for theorem proving in first order theory with equality, preprint.

[8] J. Hsiang and M. Rusinowitch, On word problems in equational theories, in Proc. 14th ICALP, Karlsruhe, Germany (1987) 54-71.

[9] G. Huet, A complete proof of the Knuth-Bendix completion algorithm, J. Comput. System Sci. 23 (1981) 11-21.

[10] G. Huet and D. C. Oppen, Equations and rewrite rules: a survey, in: R. V. Book, Ed., Formal Language Theory, Perspectives and Open Problems (Academic Press, New York, 1980) 349-405.

[11] A. Kandel, Fuzzy Mathematical Techniques with Applications (Addision-Wesley, Menlo Park, CA, 1986). 
[12] D.E. Knuth, and P.B. Bendix, Simple word problems in universal algebras, in: J. Leech, Ed., Computational Problems in Abstract Algebras (Pergamon Press, Oxford, 1970) 263-297.

[13] R.C.T. Lee, Fuzzy logic and the resolution principle, J. ACM 19(1) (1972) 109-119.

[14] C.J. Liau and I.P. Lin, Fuzzy logic with equality, Internat J. Pattern Recognition and Artificial Intelligence 2(2) (1988) 351-365.

[15] G.E. Peterson, A technique for establishing completeness results in theorem proving with equality, SIAM J. Comput. 12 (1983) 82-100. 